\title{
Ludwik Flecks Gestaltbegriff und sein Blick auf die Gestaltpsychologie seiner Zeit
}

\author{
Claus Zittel
}

Ludwik Fleck and the Gestalt psychology of his time

The notion of ,Gestalt' plays a prominent role in Ludwik Fleck's theory of thought styles. This paper scrutinizes how Fleck adopted the concepts and even methods of Gestalt psychology that he sometimes vaguely refers to. Systematically comparing the argumentation and theoretical outlines of Fleck's social theory of perception and the principles of some Gestalt theories, this article will show and discuss their similarities and fundamental differences. According to Fleck, both science and individual perception rest on social actions and cultural traditions. In particular, Fleck emphasized the relevance of the common language and collective meanings for the shaping of objects according to the thought style of a scientific collective. In contrast to the principles of Gestalt theories, in Fleck's view not only the perception, but also the constitution of scientific objects and even the ,laws' of perception depend on social and cultural constructions of reality. That leads him to a particular theory of Gestalt perception.

Keywords: Ludwik Fleck, Gestalt psychology, social construction, perception, thought styles

Schlüsse/wörter: Ludwik Fleck, Gestaltpsychologie, Gestaltwahrnehung, Soziale Konstruktion, Wahrnehmung, Denkstil

Wenn unser Sehen kein Gestaltsehen wäre, würden dann abstrakte Begriffe entstehen, wäre eine Verallgemeinerung und überhaupt Wissen möglich? (Fleck 2011: 404)

Den Kindern erklären Sie bitte, daß das Chamäleon „ZIGI“ hieß. Nicht ich habe das ausgedacht, aber es hieß so seit der Erschaffung der Welt. „Z“ symbolisiert seine groteske, zickzackförmige Silhouette, zwei „I“ die Metamystik seiner Gestalt, und der Buchstabe „G“ steht für seine löffelförmige Zunge, mit der es blitzschnell die Fliegen fängt. Es ist ein sehr interessantes und mystisches Geschöpf. (Fleck 2011: 601)

\section{Gestaltpsychologische Ansätze bei Fleck?}

Zweifellos spielen der Begriff der Gestalt und das Thema der Gestaltwahrnehmung bei Fleck eine zentrale Rolle. ${ }^{1}$ Die konkreten Bezugnahmen auf die 
Gestaltpsychologie sind jedoch selten und trotz entsprechender Bildbeispiele sehr allgemein gehalten. Es wird in diesem Beitrag also zu klären sein, welche gestaltpsychologischen Ansätze Fleck im Einzelnen rezipierte und welche Funktion und Relevanz diese Bezüge für seinen Gestaltbegriff haben konnten. Zugleich werde ich der Frage nachgehen, inwiefern sich systematische Vergleichspunkte zwischen Flecks Denkstiltheorie und diversen Gestalttheorien finden lassen. Eine Hauptschwierigkeit dabei ist, dass sich die so genannte Gestaltpsychologie nicht als einheitliche Schule präsentiert, sondern sehr verschiedene und damit schwer zu überschauende Varianten gestaltpsychologischer Ansätze ins Spiel kommen. Wie breit und facettenreich das Spektrum dieser Ansätze ist, haben das ausgezeichnete Standardwerk von Mitchell Ash (1995/2007), aber auch seine konzise Darstellung zu Wertheimer und Köhler (Ash 2009) sowie unter anderem der von Smith (1988a) herausgegebene Sammelband und die Überblicksdarstellungen von Herrmann (1976) und Fitzek/Salber (1996) vorgeführt. Fleck wird in Arbeiten zur Geschichte der Gestalttheorien wenn überhaupt (Harrington 1996/2002), dann nur am Rande erwähnt (Ash 1995/2007: 219, Smith 1994: 9 und 18), wobei seine Position weder geklärt noch historisch im Kontext der gestaltpsychologischen Strömungen genauer verortet wird, da sie offenkundig für diese keine nennenswerte Rolle gespielt hat. Auch die spärliche Forschungsliteratur, die explizit nach der Rolle der Gestaltwahrnehmung bei Fleck fragt, rekonstruiert nicht, was Fleck überhaupt an Vorarbeiten kannte und wie sich seine Denkstillehre systematisch $\mathrm{zu}$ diversen Positionen gestaltpsychologischer Provenienz verhält (Hagner 2010, 2012, Löwy 2008). Zuweilen wird eher unbestimmt eine Verwandtschaft der Konzepte (Freudiger 2010: 7) erklärt, manchmal auch eine methodische Nähe im Hinblick auf den experimentalwissenschaftlichen Forschungsansatz (Stahnisch/Bauer 2007: 11) behauptet. Michael Hagner konstatierte sogar unter Berufung auf Flecks Selbstaussagen, dieser habe „einen ganzheitlichen oder holistischen Ansatz“ verfolgt, „den er der Wahrnehmungspsychologie entlehnt“ (Hagner 2010: 584). Hagner muss jedoch in der Fußnote dazu einzuräumen, dass bis heute nicht klar sei, um welche Quellen es sich handelt (ebd.: 494, Fußnote 21, vgl. auch Hagner 2012: 113). Wie insbesondere auch aus Ashs Arbeiten hervorgeht, ist jedoch nicht jede Theorie der Wahrnehmung von Gestalten eine gestaltpsychologische Theorie und Fleck könnte hier einem Selbst-Missverständnis erlegen sein, das der Vieldeutigkeit des Gestaltbegriffs geschuldet ist. Es kann im Rahmen dieses Artikels indes nicht darum gehen, einen Überblick über die verschiedenen gestaltpsychologischen Ansätze zu geben, sondern lediglich darum, zu fragen, was Fleck kannte, was er kennen konnte und was eine systematische Konfrontation der jeweiligen Positionen ergäbe. Weder vonseiten Flecks noch vonseiten der Vertreter und Historiker der Gestaltpsychologie ist diese Diskussion geführt worden. Führt man sie nachträglich, so zeigt sich überraschenderweise, dass sich Flecks Ansatz fundamental von 
jenen ihm mutmaßlich bekannten Gestalttheorien unterscheidet und als eigenständiger, alternativer Entwurf einer Theorie des Gestaltsehens begriffen werden kann. Flecks Vorstellung, wie Gestaltsehen funktioniert, verdankt sich somit weniger der Entlehnung gestalttheoretischer Ansätze und Konzepte, sondern hat aus diesen nur einzelne Bruchstücke herausgeklaubt und der eigenen Konzeption einverleibt.

\section{Was zitiert Fleck und wie?}

Fragt man nach konkreten Zeugnissen einer Rezeption gestaltpsychologischer Theorien bei Fleck, so ist die Antwort schnell zur Hand: Der einzige explizite Hinweis, den Fleck gibt, findet sich in einer Fußnote zu seinem Hauptwerk Entstehung und Entwicklung einer wissenschaftlichen Tatsache (Fleck 1980 [1935]: 39: Fußnote 4), und dieser bezieht sich auf ein Sammelreferat von Wolfgang Metzger, das die Gestalttheorie in der Musikpsychologie Erich von Hornbostels vorstellt (Metzger 1929). ${ }^{2}$ Fleck zitiert aus dem Metzger-Referat nur aus einem Abschnitt, der mit „Laut und Sinn“ überschrieben ist. Im Lichte von Humboldts Ursprungstheorie der Sprache diskutiert Metzger hier Überlegungen Hornbostels etwa zur Frage, wie in der Lautmalerei Erlebnisse in unmittelbar sinnvolle Laut-Gebilde übertragen werden. In diesem Falle erfolge keine konventionalistische Zuordnung von Zeichen zu Bezeichnetem, sondern es bestehe eine unmittelbare Ausdrucksrelation. Fleck zitiert den entsprechenden Passus ausführlich und gleich zweimal den Satz, es handle sich hierbei um „eine Übertragung der Erlebnisse und Gegenstände in ein Material, das leicht formbar und stets bei der Hand“ sei (Fleck 1980 [1935]: 39).

Der Zitat-Kontext klärt darüber auf, dass Fleck im Metzger-Referat offenbar ein Konzept für eine Erklärung der sinngerichteten Verwandlung von Lautgebilden vorfindet, das er benutzen kann, um seine Vorstellung einer beständigen Transformation von Denkgebilden, insbesondere der von ihm so genannten Präideen, anschaulicher und plausibel zu machen. Denn es ist nicht einfach nachzuvollziehen, dass der vage Sinn dieser Prä- oder Urideen durch Jahrhunderte persistent bleibt, obwohl diese Ideen permanent mutieren und in verschiedenen Epochen zu Gestalten reifen, die in ganz unterschiedlichen Kontexten jeweils unmittelbar evident erscheinen und einen magischen Zauber ausstrahlen können. Unmittelbar vor und nach dem Zitat stellt er jedoch durch ein zweifaches „vielleicht“ klar, dass es sich hier um ein Gedankenspiel handelt:

Vielleicht erläutert besser die Bedeutung der Präideen ein anderer Vergleich, aus dem Gebiete des neuerlich von Psychologen vermuteten Ursprungs der Worte [...] Die Tatsache der Präideen läßt vielleicht auf ähnliche Verhältnisse schliessen. (Ebd.) 
Fleck spielt dann durch, wie Hornbostels Gedanke sich auf seine Konzeption auswirken würde, das heißt er zeigt an seiner eigenen Idee, wie eine solche Übertragung ablaufen könnte und wie sein Konzept dabei umgeformt würde. Dafür tut er nichts anderes, als Metzgers Zitat Satz für Satz umzuwandeln:

Gedankliche Wiedergabe wäre ursprünglich nicht eindeutige Zuordnung im Sinne der Logik, sondern eine Übertragung der Erlebnisse in ein Material, das leicht und stets formbar bei der Hand sei. Der Zusammenhang zwischen Wiedergabe und Erlebnissen gliche nicht dem konventionellen Verhältnis zwischen einem Zeichen und dem Bezeichneten, sondern läge in der psychischen Entsprechung beider. Die Evidenz wäre in derart entstandenen Denkgebilden unmittelbar enthalten. (Ebd.)

Die Schlussfolgerungen aus dieser hypothetischen Umformung Hornbostels zieht Fleck allerdings dann im Indikativ und ungeachtet dessen, dass Metzger in seinem Referat direkt im Anschluss an die zitierte Passage das Scheitern von Hornbostels Hypothesen konstatierte. ${ }^{3}$

Ideen würden demnach permanent in andere Bereiche übertragen und dabei umgebildet, was deshalb möglich sei, weil Worte und Ideen ursprünglich keine Namen für Gegenstände in der Welt seien, sondern

ursprünglich lautliche und gedankliche Äquivalente der Erlebnisse, die gleichzeitig mit ihnen gegeben sind. Dies erklärt die magische Bedeutung der Worte und die dogmatische, religiöse Bedeutung der Sätze. Parallel der Bedeutungsentwicklung der Worte nach Hornbostel gibt es auch eine Ideenentwicklung, die nicht etwa durch Abstraktion vom Besonderen zum Allgemeinen, sondern durch Differenzierungen (Spezialisierung) vom Allgemeinen zum Besonderen geht. (Ebd.) ${ }^{4}$

Es wird nun jedoch gerade in einem Passus aus Metzgers Referat, den Fleck übergeht obwohl er das Verbindungsstück der beiden von ihm wörtlich zitierten Stellen ist, deutlicher, wie die „parallele Bedeutungsentwicklung“, auf die Fleck hinaus will, zu verstehen sei:

Natürlich sind für eine solche Untersuchung Sprachen sehr früher oder sehr primitiver Völker am ergiebigsten; aber es ist doch erstaunlich, wieviel unmittelbar sinnvolle Gebilde auch die europäischen Sprachen noch heute enthalten, trotz des verzerrenden Einflusses der Lautverschiebungen und der schriftlichen Überlieferung und trotz des stets wachsenden Bestandes an willkürlichen, „sinnlosen“ Bezeichnungen. [...] Wie v. Hornbostel an vielen Beispielen nachweist, bedeuten die primitiven Lautgebilde stets sehr viel auf einmal, nämlich immer sämtliche einander entsprechenden Inhalte aller möglichen Wahrnehmungs- oder Seinsgebiete. Es ist nicht so, daß eine Bedeutung in einem Seinsgebiet entstanden und nachträglich auf andere „übertragen“ worden wäre. (Metzger 1929: 846)

Erst auf der Basis dieses Befunds wird Flecks Analogiebildung plausibel, nämlich, dass bei den ursprünglichen Worten der Sinn der Urideen nicht durch eine Relation zu einem Gegenstand oder eine konventionelle Festlegung feststand, sondern eine Idee oder ein Wort zugleich verschiedenen psychischen Erlebnissen korrelieren und dann dennoch als Komplex unterschiedlichster Erlebniswelten tradiert werden konnte. Dies ist nicht zuletzt deshalb wichtig, weil von dieser Konzeption aus ein klärendes Licht auf 
den Polyperspektivismus in Flecks Denkstiltheorie fällt, das insbesondere seine Vorstellung erhellt, welche Transformationen auch wissenschaftliche Termini im intrakollektiven Denkverkehr erfahren können. Denn so tritt zutage, dass dieser Denkverkehr auch dann funktioniert, wenn die Einzelnen ein gemeinsam verwendetes Wort verschieden verstehen. Später, 1939, greift Fleck das gleiche Zitat wieder auf und wandelt es ab:

Anschauungen entstehen als Transpositionen von Erlebnissen innerhalb eines sehr plastischen Materials, welches sich leicht von einem Mitglied des Kollektivs auf ein anderes übertragen läßt: in Worten. (Fleck [1939] 2011: 324)

Als eine entscheidende Differenz zur zitierten Quelle zeigt sich hier, dass Fleck die prägende Rolle der kulturellen Sprachkonventionen und Vorurteile nicht nur bei der Wahrnehmung von Objekten betont, sondern dass er die Objekte selbst als bereits durch die Sprache und Tradition konstituiert begreift. Diese Gegenstände sind daher keineswegs neutral gegeben, weshalb dann auch nicht im Anschluss deren Wahrnehmung unabhängig von diesen sprachlichen Konstituenten erforscht werden könnte:

Den überwiegenden Teil unserer Gestalten (obwohl wahrscheinlich nicht alle) hat die Umgebung geschaffen, die Sprachgewohnheit, die Meinung der Allgemeinheit, die Tradition. Sie dressieren uns auf eine gewisse Ganzheit: Das Kollektiv gibt die Sanktion, gewisse Ganzheiten aus einem Komplex gewisser Elemente abzusondern. Es schafft einen Begriff von gewissem Inhalt und gewissem Bereich, und dieser Begriff realisiert sich, wird Körper, Gestalt mit gewissen Merkmalen und gewissem Bereich an Transpositionen. Und wer Mitglied des Kollektivs ist, sieht sie. (Fleck 2011 [1939]: 394-404, hier: 399)

Und:

Der Forscher erschafft auch Transpositionen für seine Erlebnisse, nur sind seine Methoden und sein Material noch mehr mit einer spezifischen (wissenschaftlichen) Tradition verbunden. (Fleck 2011 [1939]: 358)

Der hier nun verwendete Ausdruck „Transposition“ für solche Übertragungen findet sich weder im Metzger-Referat noch im Kontext seiner Zitation bei Fleck, es muss folglich noch eine andere Quelle geben. Der aktuelle Kontext in „Schauen, Sehen, Wissen“ verweist auf Christian Ehrenfels und Alexius Meinong ${ }^{5}$, die den Transpositionsgedanken so erläutern, dass die Gestalt einer Melodie als Ganzes eine andere Gestaltqualität besitze als die Gestalt ihrer Elemente (Töne), somit die Melodie in eine andere Tonart transponiert werden könne, und dennoch erhalten bleibe, solange die Beziehungen zwischen den Elementen mit transponiert werden (vgl. Ehrenfels 1890, dazu Ash 1995/2007: 171-173). Genau dieses Beispiel führt Fleck an und ich vermute, dass er zumindest Ehrenfels' Aufsatz über die Gestaltqualitäten kannte. Da Ehrenfels zufolge nicht nur melodische, sondern auch harmonische Verbindungen transponierbar sind, präludiert hier zudem Flecks Vorstellung einer „Harmonie der Täuschungen“ (Fleck 1980 [1935]: 40-53, 
Fleck 2011 [1946]: 385), die eben auch dann sich einstellt, wenn oszillierende Bilder sich zu einer evidenten Gestalt arretieren.

Wie dem auch sei, diese und die obigen Zitate zeigen, dass Fleck sich primär für den Mechanismus der Transposition interessiert, aber nicht für die theoretische Grundlegung, die die Gestaltpsychologie bei Wertheimer, Köhler, Ehrenfels und Meinong und nur ferner von Hornbostel und Metzger, erfahren hatte. Dies mag erklären, warum er hier keine Nachweise bringt, sondern nur allgemein bekundet, die „Psychologie“ lehre

daß jede Wahrnehmung vor allem das Sehen irgendwelcher Ganzheiten ist, man aber ihre Elemente erst danach sieht. [...] Eben genau solche Ganzheiten, die sich den sinnlichen Wahrnehmungen direkt aufdrängen, in hohem Maße unabhängig von ihren Bestandteilen, nennt die Psychologie „Gestalten“, unabhängig vom Sinn, der sie vermittelt. Also kann es visuelle Gestalten geben, z.B. ein Kreuz, ein Buchstabe, eine Ziffer, oder auditive, z. B. eine bestimmte Melodie, ein Wort, oder Geruchsgestalten, z. B. der Geruch von Gewürzläden, der Geruch von Bahnhöfen. (Fleck 2011 [1947]: 392)

Auch bei diesem allgemeinen Hinweis kann man beobachten, das Fleck keine Aussage über den Status der zu sehenden Ganzheiten macht, sondern lediglich einen Terminus übernimmt, ohne dabei auf die gestaltpsychologischen Erklärungen der Ursachen und Funktionen jener Ganzheitsgestalten einzugehen. Doch immerhin bringt er eine Handvoll Bildbeispiele gestaltpsychologischer Provenienz. Hierbei ist wiederum auffällig, dass deren spektakuläre Kippbilder-Beispiele (Hornbostel 1922) ${ }^{6}$ - wie die Rubin-Vase (Rubin 1921) - nicht bei Fleck vorkommen, sondern eher am Rande erwähnte Phänomene wie die Ergänzung von Teilelementen zu Ganzheiten anhand der Wahrnehmung von Buchstaben (Köhler 1928) (Abb. 1).

Der Grund dafür ist wohl, dass bei einem Kippbild das Auge entweder die eine oder die andere Gestalt sieht, die jeweilige Gestalt dann aber immer klar

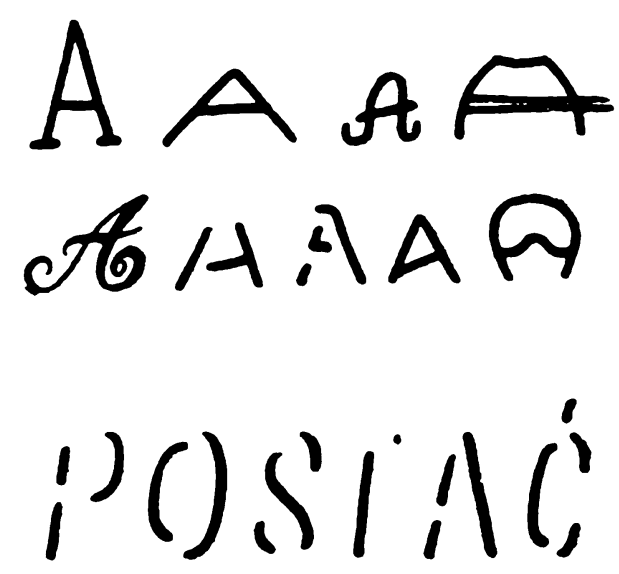

Abb. 1 Transposition von Buchstaben, aus Fleck 2011 [1947]: 395) ${ }^{7}$ 
definiert ist, entweder sieht man eine Vase oder ein Gesicht, einen Hasen oder eine Ente, die Form ist dabei vorgängig fix definiert. Fleck interessiert indes, wie sich solche Formen erst in kollektiven Sehprozessen gemäß den gegebenen kulturellen und sozialen Umständen herausbilden, dabei nicht nur zwei, sondern viele verschiedene transitorische Gestaltungen erfahren können, die für kürzere oder längere Zeit fixiert werden. Fleck führt daher stattdessen viele Bildbeispiele an, die in der Gestaltpsychologie keine Rolle spielen, medizinische Abbildungen aus der frühen Neuzeit etwa oder eben Mikroskopbilder. Flecks historische Bildbeispiele, die zeigen sollen wie Bilder aus der Tradition so lange umstilisiert werden, bis sie eine Gestalt erhalten, die zu einem neuen Denkstil passt, ${ }^{8}$ stammen meist aus Sudhoffs einschlägiger Studie über medizinische Illustrationen (Sudhoff 1907). ${ }^{9}$ Fleck bringt die gleichen Abbildungen mehrfach, das spricht nicht dafür, dass er in diesem Bereich (anders als in der Mikroskopie) einen größeren Bildfundus vor Augen gehabt hätte.

Dass es ausgerechnet die Buchstabenbeispiele sind, anhand derer Fleck meint „die Frage des Gestaltsehens [...] am besten“ (Fleck 2011 [1947]: 392) darstellen zu können, rührt offenbar daher, dass konventionell in der Serologie die unter dem Mikroskop beobachteten Bakterien Buchstabenformen beigelegt bekommen. Fleck selbst verwendet diese Buchstabenvergleiche in seinen medizinischen Schriften, zum Beispiel zur Beschreibung der Streptokokkenvariationen oder der Diphtheriebazillenformen und macht dabei klar, dass diese Bakterien so nicht aussehen, sondern ihre Gestalt kollektiv erzeugt wird. So könne eine „Bazillenkolonie das beschriebene Aussehen gar nicht besitzen“, dennoch sei

ihr Aussehen spezifisch und für ein geübtes Auge sofort erkennbar, ähnlich wie wir den Buchstaben A erkennen, auch wenn seine Proportionen sowie die Stilisierung sehr verschieden sind. (Ebd.: 614) ${ }^{10}$

Fleck führt dieses Buchstabenbeispiel immer wieder an, um zu illustrieren, wie eine stilgemäße Determination der Wahrnehmung durch Kollektive funktioniert, er setzt es also für spezifische Zwecke ein, die sich grundlegend von den diversen Erklärungszielen der Gestaltpsychologie unterscheiden. Um dies genauer erläutern zu können, muss ich zunächst einige hierfür wichtige Aspekte von Flecks Denkstiltheorie skizzieren.

\section{Gestaltsehen nach Fleck}

Fleck definiert Denkstil als einen „bestimmte[n] Denkzwang und noch mehr: die Gesamtheit geistiger Bereitschaften, das Bereitsein für solches und nicht anderes Sehen und Handeln“ (ebd.: 85). Die „Bereitschaft zum Wahrnehmen [...] spezifischer Gestalten“ ergebe „sich daraus, wie ein Denkstil entsteht: 
nämlich durch das Kreisen der Gedanken innerhalb eines Kollektivs“ (ebd.: $201)^{11}$ sowie durch kollektive Stimmungen, die für die Ausrichtung und temporäre Verdichtung der Wahrnehmung zu Gestalten sorgen (ebd.: 287) und durch die subkutan wirkende kulturelle Traditionen von Praktiken und Ideen prägenden Einfluss auf die Wahrnehmungsgestalten erhalten:

Wenn wir nun die wissenschaftliche Entdeckung beschreiben als das Wahrnehmen einer neuen Gestalt, einer neuen Ganzheit oder eines neuen Inhalts, immer dort, wo es bislang nur sinnloses Chaos gegeben hat, dann ist ihre Entstehung auf zwei Wegen möglich gewesen: entweder (1) als ein Ausdruck im wissenschaftlichen Stil irgendeiner mehr oder weniger unklaren Präidee, die spontan entstand, oder (2) auf dem Weg der beschriebenen Verdichtung irgendeines Gedankens aufgrund seiner Wanderung innerhalb eines Kollektivs. (Ebd.: $201 \mathrm{f}$.)

Wie und was für eine Gestalt wahrgenommen wird, hängt also davon ab, welche Wirklichkeit eine soziale Gemeinschaft aufgrund spezifischer Stimmungen und gemäß eines besonderen Stils geschaffen hat. Hierbei verknoten sich diachrone Ideenlinien gleichsam mit synchronen Netzen, werden zum Ausgangspunkt neuer Linien, neuer Verknotungen, die wiederum die alte Linie verändern, „immer neue Knoten entstehen und die alten Knoten verschieben sich gegenseitig. Ein Netzwerk in fortwährender Fluktuation: es heißt Wirklichkeit oder Wahrheit" (Fleck 1980 [1935]: 105). Es gibt somit viele Wirklichkeiten, die jeweils von sozialen Gemeinschaften nach dem in ihnen vorherrschenden Denkstil entworfen werden:

Zwischen dem Subjekt und dem Objekt gibt es ein Drittes, die Gemeinschaft. Es ist kreativ wie das Subjekt, widerspenstig wie das Objekt und gefährlich wie eine Elementargewalt. (Fleck 2011 [1960]: 470)

Die Schöpfungen des Kollektivs beschränken sich hierbei keineswegs auf das Denken, sondern erstrecken sich ebenso in den Bereich der Anschauung, ein Denkstil ist zugleich ein Sehstil. Das darf man sich jedoch nicht so vorstellen, dass alle Mitglieder eines Kollektivs durch die gleiche Wahrnehmungsbrille schauen oder in selben Denkmustern denken. Denkstile sind weder fixe Denkstrukturen noch Denkformen oder Weltanschauungen, sondern als Vorgänge aufzufassen: als Zirkulationsprozesse von Ideen und Praktiken und die aus ihnen resultierende unbewusste stilgemäße Konditionierung von Wahrnehmung, Denken und Handeln der Forscher, die ständige Transformationen erfährt (Zittel 2011). Fleck arbeitet nun an vielen Stellen en detail eine regelrechte Phänomenologie und Psychologie des wissenschaftlichen Sehens und Handelns aus. Grundsätzlich unterscheidet er drei Etappen des Erkenntnisverlaufes: In der ersten Phase zeige sich beim Beobachten von Laborpräparaten zunächst ein „Chaos widersprüchlicher, einander abwechselnder Bilder". Das bis dahin feststehende Bild zerfalle

in Kleckse, die sich zu verschiedenen, widersprüchlichen Gestalten formen. [...] Von der Stimmungsspannung des Forschers [hänge] ab, ob ihm die neue Gestalt 
als symbolische grelle Vision erscheint, oder auch als schwaches Aviso eines Widerstands, der die ungebundene, fast willkürliche Auswahl unter den sich abwechselnden Bildern bremst. (Fleck 2011 [1935]: 232)

Diese Gestalt muss dann von Unwichtigem separiert und stabilisiert werden, indem man eine Denkbereitschaft schafft und Menschen dazu dressiert, für eine gewisse Zeit das Gleiche zu sehen. Wenn dies gelungen ist, werde die Gestalt unmittelbar als evident wie „eine unabhängige, einzige, ewige Wahrheit wahrgenommen" (ebd.).

Fleck hebt hervor, dass wir die Sprache einsetzen müssen, um überhaupt erst den Beobachtungsgegenstand als solchen in den Blick bekommen zu können, der also keineswegs bereits in bestimmter Gestalt vorliegt oder nur durch die Anschauung gegeben wird und dann ,neutral' beschrieben werden könnte. „Der Satz: Jan hat das Phänomen P erkannt, ist unvollständig, man muß ihm hinzufügen: im Denkstil S, eventuell auch in der Epoche E“ (ebd.: 284). Es gebe keine einfachen Beobachtungen, die durch Protokollsätze erfasst werden könnten, erst recht nicht könnten diese Protokollsätze dann als Fundament für den Aufbau der Wissenschaften dienen, wie es Carnap vorschwebte. Die Worte reichen einerseits nie aus, um das jeweilige Beobachtungserlebnis adäquat einzufangen, andererseits bedarf man ihrer, um die Beobachtung so $\mathrm{zu}$ stilisieren, dass man überhaupt etwas erkennt. Zunächst muss man dazu eine Reihe suggestiver Fragen stellen, wie beispielsweise: Was ist hier Vordergrund, was Hintergrund? Ist es

eine Sammlung dunkler Striche auf hellem Hintergrund, [...] irgendeine zufällig aus jenen Strichen gefügte Figur, z. B. eine zerklüftete Insel? (Sind die Striche in ihrer ganzen Länge gleich stark? Sind sie gerade? Färben sie sich homogen? Liegen sie parallel? usw.). (Ebd.: 216 f.)

Beobachte man ein mikroskopisches Präparat, etwa mit Diphtheriebazillen, so habe man:

(wie es scheinen könnte) nur eine Anzahl gefärbter kleiner Striche von besonderer Form, Struktur und Lagerung vor sich, die zusammen ein charakteristisches Bild geben. Vergeblich wäre die Bemühung, mit bloßen Worten das Charakteristische des Bildes zu beschreiben, das der Fachmann sofort sieht, das vom Laien aber zunächst nicht erkannt wird. Diesen muß man erst vorbereiten: durch Gleichnisse, die ihm bekannte Figuren enthalten und durch Vergleiche mit anderen mikroskopischen Bildern, schließlich durch Gewöhnung an das Zuordnen entsprechender fachtraditioneller Worte; man muß eine gerichtete Bereitschaft für das Wahrnehmen dieser besonderen Gestalt erwecken, die die Wissenschaft schuf. (Ebd.: 250 f.)

Erst allmählich entwickelt sich gemeinsam mit dem Denkstil eine stilgemäße Wirklichkeit. „Sehen“ heißt für Fleck „im entsprechenden Moment das Bild nachzubilden, das die Denkgemeinschaft geschaffen hat" (ebd.: 233). Eine Beobachtung wird jedoch nicht allein durch Sprache stilgemäß determiniert, sondern auch Instrumente und Apparate treten hinzu und zwingen 
gemeinsam mit den Beschreibungen dem Beobachter einen bestimmten Sehstil auf. Wissenschaftliche Bilder tragen dazu bei, aus der Beobachtung fiktive Entitäten herauszumodulieren, die aufgrund ihrer anschaulichen Evidenz als tatsächliche Objekte erscheinen. Die in Fachzeitschriften und später in Schulbüchern publizierten Bilder führen schließlich dazu, dass die Forscher zu glauben beginnen, zum Beispiel ein Bazillus oder eine Darmwindung sehe tatsächlich so aus, - ihr mit viel Aufwand manipuliertes Bild wird zur Tatsache.

Fleck selbst hat anlässlich eines Vortrags die Konzeption seiner Gestalttheorie prägnant zusammengefasst, sie sei daher ausführlich zitiert:

Der Vortragende versucht anhand von Beispielen aus der Geschichte der Anatomie (durch das Zeigen von Abbildungen und eine Analyse der Beschreibungen), aus der Geschichte der Medizin (Uroskopie) und an einem sehr instruktiven Beispiel aus der Mikrobiologie (anhand der Frage der Bakteriengeißeln in der klassischen Bakteriologie und den neusten Beobachtungen von Pijper) aufzuzeigen, daß jede wissenschaftliche Beobachtung gemeinschaftliche Vorstellungen einer Epoche und einer Umwelt enthält. Es gibt eine gerichtete Wahrnehmungsbereitschaft, die dem Sehen unmittelbar das Wahrnehmen von ganzheitlichen Gestalten aufzwingt. Diese Gestalten nehmen dadurch den Charakter von unabhängig existierenden Dingen an.

Gäbe es nicht das Gestaltsehen, wären wahrscheinlich sowohl das Bilden von abstrakten Begriffen als auch das Verständigen - also generell die Wissenschaften - unmöglich. Die sogenannten Naturwissenschaften (Physik) erzeugen durch den Prozeß der Analyse und durch das Fingieren von idealen Gegenständen spezifische Gestalten, die man dank einer durch das Zusammenleben innerhalb einer bestimmten Gruppe entstandenen, spezifischen Wahrnehmungs- und Assoziationsbereitschaft sieht und handhabt.

Die wissenschaftliche Apparatur und die Berechnung modellieren eine Gestalt aus dem Chaos der Empfindungen, natürlich nicht wie es einem Individuum erscheint, sondern im Geiste des Denkstils einer Wissenschaft in einer Periode.

Das Erkennen und insbesondere die Beobachtung ist eine Funktion mit drei Gliedern: Neben dem sogenannten Subjekt und dem sogenannten Objekt hat an der Beobachtung die jeweils gegebene Gemeinschaft Anteil, die ich als Denkkollektiv bezeichne. Die Denkkollektive besitzen einen spezifischen Denkstil, eine spezifische soziologische Struktur und eine spezifische historische Entwicklung. (Ebd.: 534)

\section{Ähnlichkeiten und Differenzen}

Wir sind nun an einem Punkt angelangt, an dem wir ausreichend Informationen gesammelt haben, um einen kontrastierenden Vergleich durchführen zu können: Fleck greift zwar einzelne Motive wie die Transposition auf und zuweilen scheint seine schöpferische Gestaltkonzeption auch eine Nähe zur Produktionstheorie der Grazer Schule aufzuweisen (vgl. Anm. 13), der zufolge Wahrnehmung als aktives Geschehen $\mathrm{zu}$ verstehen ist, das sinnhaft 
strukturierte Komplexionen herbeiführe. Bei der Gestaltproduktion kämen auch Erfahrung, Training und innere Einstellungen insbesondere dann ins Spiel, wenn kein klares Reizmaterial vorliegt (Koffka 1914, 1919b, Sander 1928). Friedrich Sander wiederum ist der „Zweiten Leipziger Schule“ der Gestaltpsychologie $^{12}$ zuzurechnen, die sich kritisch mit der „Berliner Schule“ auseinandersetzt und insbesondere die Rolle der Gefühle bei der Entstehung von Wahrnehmungsgestalten und somit die individuelle Prozessualität dieses Vorgangs herausarbeitet (Sander 1928). In Flecks Unterscheidung mehrerer Phasen in der Gestaltwahrnehmung scheint hier also ein verwandtes Motiv anzuklingen, auch wenn offen bleiben muss, ob er Sanders Arbeiten gekannt hat. Flecks Rede vom „Widerstandsaviso“ erinnert zudem an Begriffe aus der Gestaltpsychologie wie „Vorgestalt“, dem „rauschhaften Schock“ (Sander 1928: 58-59) und dem „Aha-Erlebnis“ beim plötzlichen Erkennen eines gedanklichen Zusammenhangs (Bühler 1907), oder auch an Meinongs Unterscheidung zwischen Ernstgefühlen und Scheingefühlen, ${ }^{13}$ bei der ja letztere keineswegs als Phantasmata, sondern als reale innerpsychische Phänomene anzusehen seien. Bei Fleck taucht diese Abstufung zwischen Phantasie-Gefühl und bona fide-Gefühl auf, wenn er die Phase beschreibt, in der ein Forscher noch unsicher ist, ob das was ihm gegenüber steht, ein reales Gebilde oder sein Phantasiekonstrukt ist. Nun trifft er auf ein Widerstandsaviso, und nach und nach stabilisiert sich der Eindruck der Festigkeit des Gegenstandes und damit korrelierend das Objektivitätsgefühl, der Gegenstand erscheint als real existierend (vgl. Ingarden 1964/1965, Brod/Weltsch 1913). Weiter kommt man Fleck zufolge jedoch nicht. ,Wirklichkeit' ist hier nicht als Welt da draußen zu verstehen, an die man stößt, sondern es werden seitens der Forscher Eindrücke verdichtet und ihre Sehnsucht nach harter Realität, der gegenüber sie sich passiv verhalten können, lässt sie diese Verdichtungen als Widerstand erfahren. Einer der großen Vorzüge von Flecks Konzeption ist, dass er ohne ontologische Hypostasen auskommt und dass sich für ihn das Problem eines Beweises für die Realexistenz von bewusstseinsexternen Gegenständen, mit dem sich Intentionalitätstheorien und ihre Erben in der Gestaltpsychologie herumplagen, nicht stellt.

Trotz dieser Ähnlichkeiten gibt es jedoch weit mehr gravierende Differenzen, bei deren Darstellung es mir im Folgenden nicht darum geht, die eine gegen die andere Position auszuspielen, sondern die Verschiedenheit der Argumentationsweisen und Erkenntnisinteressen hervortreten zu lassen: Im Unterschied zur neueren Gestaltpsychologie will Fleck nicht erklären, wie die Wahrnehmung funktioniert, sondern wie Wissen entsteht. Auch wenn beim Gestaltsehen Fleck zufolge physiologische und erkenntnispsychologische Faktoren eine Rolle spielen, wird die Gestaltwahrnehmung bei ihm kulturalistisch, ästhetisch und soziologisch begründet. Er interessiert sich hierbei für das Verhältnis von ungerichteter Wahrnehmung, gezielter Beobachtung und Theoriebildung in der Wissenschaft. Seine Grundintention ist, dass sich die 
Erkenntnisweisen: diffuse Wahrnehmung, gerichtete Beobachtung und Theorie in der Praxis nicht als Abfolge verschiedener Erkenntnisstufen darstellen lassen, sondern dass sich alle drei vermischen, wobei zwar beim Vermischen wieder verschiedene Etappen unterschieden werden können, doch schon auf der ersten Stufe kulturelle und theoretische Vorannahmen die Wahrnehmung determinieren. Es gibt für Fleck daher keine einfachen Gestaltbeobachtungen, die die neutrale Basis für weitere Folgerungen bieten könnten: Gestaltwahrnehmung und Begriffsbildung spielen stets ineinander.

Fleck sucht sich nun aus ganz unterschiedlichen Gebieten Ideen zu dieser Thematik zusammen, es gibt daher eine bunte Mischung von Quellen, aus denen er für seine eigene Gestaltauffassung schöpft, darunter sind: Diskussionen um die Rolle der Beobachtung in der Quantenphysik (Bohr 1928, Fleck 2011 [1929]: 58, [1935] 219, [1947] 411), vergleichende Anatomie (Bethe 1928, Petersen 1928) und biologische Umwelttheorien (Uexküll), ${ }^{14}$ Diskussionen um Photogramme (Pijper 1930, 1931, 1938, Fleck 2011 [1948]: 534) und wissenschaftliche Bildsuggestionen Ernst Haeckels (Fleck 1980 [1935]: 51), graphische Darstellungen von Ideen, visuelle Kollektivvorstellungen in der Ethnologie (Fleck 1980 [1935]: 181-186, Fleck 2011 [1935]: 243, Lévy-Bruhl 1926: 137 f.) ${ }^{15}$ und Psychologie (Frostig 1929, Frostig 1930), Sinn-Sehen in der Geschichte der naturkundlichen Abbildung (contra Sudhoff), die Klecksographie (Fleck 2011 [1935]: 214, [1947] 405), ${ }^{16}$ sinnesphysiologische Diskussionen um mouches volantes ${ }^{17}$ (ebd.: 215) - sprachtheoretische Überlegungen zu lautlichen Gestaltqualitäten (Ingarden 1976: 44, 74 f., 104) ${ }^{18}$ - und eben die Gestaltpsychologie. Wenn man so will setzt Fleck selbst aus verschiedenen Elementen unterschiedlicher Provenienz seinen Gestaltbegriff zusammen und je nach Kontext kann bei Gestalt mal eine fiktive Entität, die graphische Darstellung einer Idee, die Stilisierung eines Bildes, die anschauliche Evidenz eines Wahrnehmungsbildes oder aber eines Vorstellungsbildes, ein gestaltendes, strukturierendes Sehen oder ein Sehen in Gestalten und von Gestalten oder Einprägungen im kulturellen Gedächtnis bedeuten, weshalb er auch zuweilen statt von Gestalt von Gepräge (Fleck 2011: [1929] 53, 56, [1932] 134, 137, [1934] 184, [1936] 288) spricht. Hieraus folgt, dass die Gestaltpsychologie lediglich zur Beleuchtung einzelner Aspekte von Flecks Wahrnehmungskonzeption, nicht aber zu ihrer Grundlegung herangezogen werden kann. Fleck interessiert sich entsprechend nicht für die Diskussionen und Streitfragen der Gestaltpsychologie, er nutzt einzelne Motive, die er dann in ein neues theoretisches Setting importiert, in dem diese Motive und Begriffe andere Funktionen übernehmen, so dass die Differenzen die Gemeinsamkeiten mit der Gestaltpsychologie überwiegen: Fleck verbindet seinen Gestaltbegriff mit einem besonderen Stilbegriff, den die Gestaltpsychologie so nicht kennt. Wenn überhaupt von Gestaltpsychologen auf Stilkonzeptionen Bezug genommen wird, so auf Alois Riegls Untersuchungen zur Ornamentik (Rubin 1921: 34). Das hat Folgen: Gestalten sind für Fleck keine fixen Formen, 
Strukturen oder Wahrnehmungsmuster, sondern transitorische Gebilde, die kulturell erzeugt werden.

Entsprechend kennt Fleck auch keine gestaltpsychologischen „Gesetze“ diese werden von ihm auch nicht aufgegriffen, vielmehr lassen sich die von ihm beschriebenen Wahrnehmungsprozesse nicht gesetzesförmig definieren.

Sehen ist für Fleck: Sinn-Sehen, es gibt also keine vorgängige Gestaltwahrnehmung, die dann so oder so interpretiert werden könnte, sondern die erste Gestaltbildung wird bereits gelenkt durch kollektive Sinn-Unterstellungen und jedes Gestaltsehen ist kollektive Interpretationsarbeit. Entsprechend hebt Fleck hervor, dass sprachliche Beschreibung und Beobachtungen bei der Herausbildung von Gestalten zusammenspielen. Die Beobachtungssprache nimmt Einfluss auf das Resultat - die eigene Fachsprache ist den Gestaltpsychologen meines Wissens in dieser Hinsicht nie zum Problem geworden. Das gleiche gilt für die Rolle der Apparate. ${ }^{19}$ Diese stilisieren nicht nur die Beobachtung mit, sondern im Instrument selbst haben sich kulturelle und wissenschaftliche Hintergrundüberzeugungen sedimentiert, wie etwa „das Fernrohr“, das es „unmöglich mache, in den Wolken noch phantastische Geschöpfe zu sehen“ (Fleck 2011 [1936]: 298). ${ }^{20}$ Wenn in manchen gestaltpsychologischen Experimenten Apparate benutzt werden, wird die Abhängigkeit der eigenen Resultate von den Apparaten, sowie von der gewählten Beschreibungssprache meines Wissens nicht thematisiert.

Bei seinen eigenen phänomenologischen Studien zum wissenschaftlichen Gestaltsehen bringt Fleck zudem Beispiele, die sich von den einfachen Experimentalanordnungen gestaltpsychologischer Untersuchungen durch ihre Komplexität stark unterscheiden. Deren Resultate müssten sich in den Augen Flecks als künstliche wirklichkeitsfremde Abstraktionen einer scientia imaginibilis ausnehmen. Für Fleck resultiert die Gestaltproduktion aus Vorgängen, die sich über längere Zeiträume hinziehen, manchmal über Jahre, und bei denen überaus viele Faktoren hineinspielen, die jenseits des Untersuchungsfeldes der Gestalttheorien liegen, darunter die schleichenden Veränderungen von Beobachtungsberichten in wissenschaftlichen Zeitschriftenaufsätzen, populärwissenschaftliche Vereinfachungen derselben und ihre Rückwirkung auf die Wahrnehmung und Überzeugungen der Forscher.

Fleck hat einen ganz anderen Begriff von Kollektiv, das - „gefährlich wie eine Elementargewalt" - keine statistisch oder auf andere Weise exakt beschreibbare Standardgruppe ist, deren Wahrnehmung experimentell untersucht werden könnte.

Der Gestaltbegriff wird in Flecks Denkstillehre dadurch erstens historisiert und kulturell relativiert, und zwar weit radikaler, als dies in jeder gestaltpsychologischen Untersuchung der Fall sein kann, da er nicht operationalisierbare Faktoren wie soziale Stimmungen, unbewusste Konditionierungen als konstitutiv für die Formierung von Gestalten ansieht. Zweitens wird er ästhetisiert, da er meint, dass die Binnenlogik des Materials, 
so wie auch „Gemälde [...] einen Zwang eigenen Stils“ kennen (Fleck 1980 [1935]: 132). Drittens wird der Stilbegriff durch irrationale Einflussfaktoren bestimmt - kollektive Riten und Stimmungen beispielsweise, die sich nicht experimentell standardisiert reproduzieren lassen. Es gibt für Fleck nicht die Möglichkeit, von einer konkreten Beobachtungssituation zu abstrahieren, die kulturellen Bedingtheiten herauszukürzen, um zu neutralen Beschreibungen zu gelangen, was zum Beispiel die Versuchsanordnungen in den Studien Köhlers (Köhler 1928, Koffka 1929) und Metzgers garantieren sollen. Spätestens dann wenn die Gestaltpsychologie in die Phase ihrer Verwissenschaftlichung eintritt, wäre auch sie ein Kandidat für Flecks heftige Kritik am Szientismus:

Ein Grundfehler vieler Betrachtungen aus dem Gebiet der Theorie des Erkennens ist, daß (mehr oder weniger offenkundig) mit einem symbolischen epistemologischen Subjekt operiert wird, genannt „der menschliche Geist“, „der menschliche Verstand“, „der Forscher" oder einfach „der Mensch“ („Jan“, „Sokrates“), das keinerlei konkrete Lebenslage hat, grundsätzlich keinen Veränderungen unterworfen ist, selbst über Jahrhunderte hinweg, und, ungeachtet des Milieus und der Epoche, jeden „normalen“ Menschen repräsentiert. Es hat also absolut, unveränderlich und allgemein zu sein. (Fleck 2011 [1936]: 260)

In den gestaltpsychologischen Experimentalanordnungen und Theorien werden die kulturellen oder gesellschaftlichen Faktoren in ihrer epistemischen Valenz kaum je thematisch: Man untersucht die Wahrnehmungswelten des Schimpansen (Köhler 1917), der Kinder, des Erwachsenen auf paradigmatische Weise. Dabei wird angestrebt, die jeweilige Gestaltwahrnehmung entweder biologisch, physiologisch, anthropologisch oder wahrnehmungstheoretisch zu begründen - für Fleck aber ist sie denkstilspezifisch und somit nur für den jeweiligen Denkstil gültig, während sie zugleich das Sehen anderer Gestalten verunmöglicht. Es gibt in Flecks Augen daher auch keine denkstilübergreifenden Korrekturen von Täuschungen durch den Vergleich subjektiv verschiedener Wahrnehmungen. Das Verschiedenheitsbewusstsein führt bei Fleck nicht zu einer Korrektur, ${ }^{21}$ sondern zur Einsicht in die Differenz der Denkstile. Fleck bezieht sich aber ausgerechnet immer dann auf die Gestaltpsychologie, wenn er wissenschaftstheoretische Objektivitätsideale hinterfragt. Im Unterschied zum Impetus der Gestaltpsychologie hat Fleck, wenn er auf sie Bezug nimmt, die gegenteilige Absicht. Seine Verweise haben einen entlarvenden Gestus, der darauf abzielt, die Mechanismen der Fiktionalisierung in den Wissenschaften zu decouvrieren und so das Objektivitäts- und Tatsachenideal als deren imaginäres Resultat vorzuführen. Wenn er sich in diesem Zusammenhang auf die Gestaltpsychologie beruft, so ist dies nicht mehr als ein ungeschicktes Autoritätsargument. Fleck führt die Gestaltpsychologie insbesondere gegen Carnap ins Treffen (Fleck 2011 [1935]: 220, Fleck 1980 [1935]: 121), um ausgerechnet den Schluss zu begründen, dass zwei Beobachter, deren Denkstile weit genug 
auseinanderliegen, „keine gemeinsamen Beobachtungsgestände haben sondern jeder von ihnen im Grundsatz einen anderen Gegenstand beobachtet." Doch gebe es nicht nur einen Relativismus in der Beobachtung, sondern verantwortlich dafür ist letztlich, dass es grundsätzlich „unmöglich“ sei, „Beobachtungsergebnisse eindeutig zu beschreiben“ (Fleck 2011 [1935]: 220, 232). Kaum ein Gestaltpsychologe würde dies zugeben ${ }^{22}$, denn dies würde nicht nur die Bedingungen der Möglichkeit des logischen Empirismus, sondern tendenziell auch die der Gestaltpsychologie untergraben. Für Fleck gibt es hingegen Wissenschaft nur im Plural und die unübersichtlichen bunten Auffassungen innerhalb der unterschiedlichen Schulen der Gestaltpsychologie wären eigentlich ein gutes Beispiel für das was Fleck „den Streit der gedanklichen Gesichtsfelder" (Fleck 1980 [1935]: 121, Fleck 2011 [1935]: 248) nennt, der eine Verständigung zwischen den verschiedenen Kollektiven erschwert oder verunmöglicht.

Auch wenn man zum Vergleich solche gestaltpsychologischen Untersuchungen heranzöge, die die Rolle der Erfahrung beim Gestaltsehen ausdrücklich thematisieren, bliebe die trennende Kluft weiterhin tief. In diesen Studien wird zum Beispiel untersucht, wie die häufigere Einprägung von Figuren sich auf deren Wiedererkennbarkeit in komplexeren Situationen auswirkt - und das war's (vgl. Gottschaldt 1926). Kulturelle Hintergründe, Sehtraditionen, die die Wahrnehmung prägen, die Binnenlogik der Figuren, all davon wird abstrahiert. Selbst wenn die Rolle des Drills bei der Gestaltwahrnehmung etwa bei Bühler, Wertheimer und Sander (Wertheimer 1923: 332, Sander 1928: 58 f.) hervorgehoben werden, bleibt dieser Drill immer im Bereich des wissenschaftlich Kontrollierbaren und auf der Seite der Versuchspersonen. Fleck hebt hervor, dass die Forscher selbst in „Einweihungsriten“ abgerichtet wurden und sie nur unter „gewissen Vorbedingungen und gewissen Zeremonien (Aufnahmesakramente)“ (Fleck 2011 [1936]: 285) zu Mitgliedern der wissenschaftlichen Gemeinschaft geworden sind. Kein Gestaltpsychologe würde einräumen, dass seine experimentellen Resultate keineswegs objektivierbar, sondern auf der Grundlage eines von kollektiven Stimmungen, ästhetischen Zwängen und magischen Praktiken determinierten Denkstils entstanden sind und ihre Überzeugungskraft durch suggestiven Wortzauber nur innerhalb des eigenen Kollektivs entfalten. Vielmehr würden Vertreter gestaltpsychologischer Positionen darauf verweisen, dass sie ein gänzlich anderes Erkenntnisinteresse leite und Flecks Untersuchungen auf einer anderen Ebene angesiedelt seien, die die ihre nicht tangiere. Fleck wiederum, würde einwenden, dass eine solche Trennung künstlich wäre und sich dem Wunschtraum einer epistemologia imaginabilis (Fleck 2011 [1936]: 261) verdanke, die meint bestimmte Bereiche der Forschung von den sozialen und kulturellen Einflüssen freihalten zu können.

Auch ein negatives Resultat ist ein Resultat und ich gebe zu, dass ich davon selbst etwas überrascht worden bin. Flecks Gestaltbegriff hat mit den diversen 
Gestaltvorstellungen der Gestaltpsychologie seiner Zeit sehr wenig gemein. Wenn er sich auf diese Vorstellungen beruft, dann nur vage, eklektizistisch und ohne Anspruch, diesen Positionen gerecht zu werden. Bei näherem Zusehen erscheinen die Ansätze vielmehr so grundsätzlich verschieden, dass man das Konzept eines kollektiven Gestaltsehens bei Fleck nicht im Einklang mit den Prämissen der Gestaltpsychologie erklären kann, sondern es als alternativen Entwurf begreifen sollte, der zwar so nicht intendiert war, jedoch im Rahmen der Denkstiltheorie eine originelle Begründung erfuhr.

\section{Danksagung}

Ich bin den anonymen Gutachtern für die ungemein hilfreichen Hinweise und Präzisierungen zu großem Dank verpflichtet.

\section{Anmerkungen}

1 Vor allem in Flecks Entstehung und Entwicklung einer wissenschaftlichen Tatsache: „Zur Krise der ,Wirklichkeit“", „Über die wissenschaftliche Beobachtung und die Wahrnehmung im allgemeinen“, „Zur Frage der Grundlagen des medizinischen Erkennens“, „Das Problem einer Theorie des Erkennens“, „Schauen, Sehen, Wissen“ und „Das Problem der wissenschaftlichen Beobachtung". Ohne Rekurs auf die möglichen Quellen aus der Gestaltpsychologie diskutieren die Wahrnehmungskonzeption Flecks: Hagner 2010, Zittel 2012, Löwy 2008. In der erweiterten englischen Version seines Aufsatzes gibt Hagner jedoch wichtige Hinweise auf Differenzen. Er sieht es als entscheidend an, dass Fleck den von der Gestalttheorie Wertheimers aufgewiesenen Wahrnehmungsbedingungen noch einen social turn gibt, siehe Hagner 2012: 113-114, vgl. dazu dazu Zittel 2011.

2 Hornbostel zählte zur Berliner Schule der Gestalttheorie um Max Wertheimer und Wolfgang Köhler, vgl. dazu Ash 1995/2007: 203-322.

3 „Die Durchführung dieses Gedankens scheiterte immer wieder daran, dass man keine Möglichkeit sah, ihn auf etwas anderes als die sprachliche Nachbildung von Gehörserscheinungen anzuwenden, die sog. ,Lautmalerei““ (Metzger 1929: 846). Fleck zeigt nun, dass es doch eine andere Anwendungsmöglichkeit gibt, allerdings um den Preis einer erheblichen Transformation des ganzen Konzeptes.

4 Fleck zitiert hier Hornbostel nach Metzger 1929: $846 \mathrm{f}$.

5 „Ähnlich kann man eine Melodie in verschiedenen Tonarten abspielen, selbst so, daß sich alle Töne geändert haben, und dennoch bleibt die Melodie dieselbe“ (Fleck 2011: 392). Vgl. auch Meinong 1929: 281: „Melodien und Figuren können häufig derart transponiert resp. verschoben werden, daß von den ursprünglichen Ton- resp. Ortsbestimmungen auch nicht eine erhalten bleibt."

6 Zur Rolle der Kippfiguren für die Gestalt- und Raumkonzepte der Moderne siehe Simons 2007: 181-202, sowie zu ihrer Diskussion innerhalb der Gestalttheorie Ash 1995/2007: $176-181$.

7 Fleck übernimmt diese Abbildungen, worauf Sylwia Werner mich hinwies, aus dem zuerst 1946 erschienenen polnischen Original von Dembowski 1955, vgl. ebd.: 149, 160. Das polnische Wort ,Postać ' bedeutet ,Gestalt - das muss man wissen, sonst ergänzt man die Buchstabenreihe zu ,posiać', was ,säen' bedeutet.

8 „Aus dem Vorrat traditioneller Bilder paßt man bestimmte Bilder und deren Kombinationen an, verwirft darauf einen Teil, stilisiert andere um, macht gewissermaßen einen 
Kampf mit den sich abwechselnd aufdrängenden Bildern durch - bis man schließlich eine neue Bereitschaft erzeugt, d. h. die Bereitschaft, eine neue spezifische Gestalt zu sehen“ (Fleck 2011: 226 f.).

9 Anhand dieser Abbildungen, Bauchsitusbilder zum Beispiel, in denen die Darmwindungen in Schneckengestalt dargestellt sind, wendet Fleck sich kritisch gegen Sudhoffs Fortschrittsgeschichte der Medizin.

10 Siehe auch Fleck 2011: 214, 222-225, 233, 392-396.

$11,[D]$ as Vertrauen der exoterischen Kreise zum esoterischen Zentrum sowie die Abhängigkeit dieses Zentrums von der exoterischen Meinung vereinfachen und verstärken den innerhalb des Kollektivs kreisenden Gedanken, ja sie verleihen ihm erst eine spezifische Gestalt. Daß die Bereitschaft zum Wahrnehmen solcher spezifischer Gestalten letztlich ein Hauptbestandteil dessen ist, was wir als den Denkstil bezeichnet haben, ergibt sich daraus, wie ein Denkstil entsteht: nämlich durch das Kreisen der Gedanken innerhalb eines Kollektivs" (Fleck 2011: 201).

12 Auch diesen Hinweis verdanke ich den anonymen Gutachtern.

13 Siehe dazu Meinong 1968/1978, Benussi 1904, 1914. Dagegen: Koffka 1919a, 1914, Köhler 1913. Vgl. ferner Stock/Stock 2008, Smith 1994, Stachel 1999, Dąmbska 1978.

14 Vgl. Fleck 2011: 253, Bethe 1928a. Bethe trat dafür ein, den Anatomieunterricht im Studium zu reduzieren, darauf verweisend, dass den Studenten im Anatomieunterricht eine der modernen Medizin inadäquate Beobachtungsweise beigebracht werde. Man müsse vielmehr lehren ,in Vorgängen zu denken, denn der Kranke ist keine Leiche, Krankheit kein Zustand, sondern ein Geschehen" (Bethe 1928b: 2404). Auch Hans Petersen, der später eine Rezension zu Entstehung und Entwicklung einer wissenschaftlichen Tatsache schreiben wird (Petersen 1936), beteiligt sich an der Debatte (Petersen 1928), und versucht im Anschluss an Heinrich Rickert und Jakob von Uexküll, das naturwissenschaftliche Denken in Kausalitäten vom medizinischen Denken zu unterscheiden: „Die Anatomie hat es in ihrem eigentlichen Bereich mit einem Zusammenhang anderer Art zu tun. Umfang und Radius bedingen einander, aber nicht als Kausalzusammenhang, sondern als Sinnzusammenhang. Die einzelnen Teile des Körpers bilden ein planmäßiges Sinngefüge. Wir sind hier bei Uexküllschen Gedankengängen, bei dem, was nach Uexküll das Besondere der Biologie ausmacht, was sie über eine bloße Anwendung von Physik und Chemie am Organismus hinausführt. [...] Für das ärztliche Denken darf man wohl das Denken in Sinnzusammenhängen als wesentlich bezeichnen. [...] Aufgabe des Kollegs ist es, das Material zu gestalten, nicht so sehr, die Dinge auseinander abzuleiten und zu entwickeln, wie es nach dem Vorbilde des Physikers der Physiologe tut. Der Anatom steht hier neben den Architekten und den Maschinenbauern, die auch Ganzheiten, gestaltend und zerlegend, verständlich machen" (ebd.: 1873, Hervorhebung im Original). Auf diese Debatte bezieht sich auch Fleck (1980 [1935]) auf S. 51, Fußnote 19.

15 Auch den Ausdruck „Ideogramm“ übernimmt Fleck von Lévy-Bruhl, vgl. Lévy-Bruhl 1926: 137 f. Vgl. dazu auch den Beitrag von Sylwia Werner in diesem Heft.

16 „Wie verhalten wir uns, wenn wir uns zum ersten Male gegenüber einem uns unbekannten Gegenstand finden? So wie ein Kind gegenüber einem verschmierten Klecks. Es sieht in ihm den Flügel eines Vogels, die Blätter eines Baumes, eine Blume, zwei zusammengewachsene Pferde, einen Engel, mit einem Wort: anderswoher bekannte Gestalten. Diese Gestalten verdrängen sich gegenseitig, verschwinden, machen einander Platz, verändern sich, oszillieren“ (Fleck 2011: 402, Hervorhebung im Original). Vgl. dazu auch Rorschach 1921.

17 Mouches volantes sind entoptische Erscheinungen (optische Erscheinungen, die ihre Ursache innerhalb des Sehsystems haben), die durch Trübungen des Glaskörpers im Auge entstehen, sie erscheinen zum Beispiel als schwarze Punkte (Mücken), Flusen, Fädchen oder Leuchtfunken. Ihre Wahrnehmung wurde bereits früher mit dem Sehen durch das Mikroskop verglichen, vgl. Bühler 1916.

18 Vgl. beispielsweise S. 74, wo das bestimmte "Gepräge (einer Gestaltqualität)" mit dem kollektiven Milieu korreliert wird, das es konstituiert, und „z. B. die Medizinersprache“ (!) mit dem Stil eines Werkganzen in Zusammenhang gebracht wird.

19 „Wir müssen uns also mit den Physikern verständigen: Nicht nur das zur Beobachtung atomarer Phänomene unumgängliche Licht verändert diesen Gegenstand der Beobach- 
tung, sondern jede Beobachtung, auch die größte und einfachste, verlangt entsprechende Bedingungen (technische und besonders intellektuelle) und wird von ihnen in bezug auf Umfang und Inhalt beeinflußt. Es gibt keine Wahrnehmung ohne Vorbildung, ohne verwickelte geistige Arbeit, aus der erst eine selbständige beobachtbare Gestalt entsteht. [...] Sogar das bloße Zeigerablesen an physikalischen Apparaten verlangt die geschulte Fähigkeit, die Gestalten des Zeigers und der Skala abzugrenzen, als sehenswürdige Gegenstände erfassen zu können, ist also nicht eine ,einfache', sondern eine kulturbedingte Beobachtung“ (Fleck 2011: 253).

20 „Das Fernrohr zeigt z. B. den den Saturn umgebenden Ring; ein im wissenschaftlichen Denkstil erzogener Mensch begreift nicht mehr, daß man, um den Zusammenhang zwischen dem im Fernrohr gesehenen Bild und einem entfernten Planeten einzusehen, in diesem Stil denken muß. Mehr noch: Schon allein solche Begriffe des ,Planeten', des ,im Fernrohr gesehenen Bildes', der ,Entfernung' oder des ,Zusammenhangs' beinhalten diesen Stil in sich. In ein Fernrohr zu sehen und in ihm dieses Bild zu sehen (und nicht z. B. die Widerspiegelung der eigenen Wimpern) und die Disposition, von dem, was man in diesem Rohr sieht, auf das zu schließen, was ,am Himmel' ist, sind bereits Elemente des wissenschaftlichen Denkstils. Wer es versteht, in ein Fernrohr zu schauen und an den Saturn zu denken, benutzt damit allein bereits einen bestimmten Denkstil. Für ihn gibt es bereits keine andere Möglichkeit mehr: Er muß den Saturnring als von ihm unabhängige Wirklichkeit und den eigenen Denkstil als den einzig, guten' anerkennen“ (Fleck 2011: 298).

21 So Victor Benussi, der zudem auf der Basis einfacher Falluntersuchungen „experimentelle Beweise" für seine Beobachtungen vorlegt, vgl. Benussi 1914.

22 Wie Ash (1995/2007: 220) zeigt, stellt hier die Position Koffkas (1925) eine Ausnahme dar, da dieser anerkennt, dass ein und derselbe Gegenstand unter verschiedenen Bedingungen verschieden gesehen werden kann.

\section{Literatur}

Ash, Mitchell, 1995 [Neuauflage 2007]. Gestalt Psychology in German Culture 1890-1967. Holism and the Quest for Objectivity. Cambridge: Cambridge University Press.

Ash, Mitchell, 1994. Gestalttheorie und logischer Empirismus. In: Lutz Danneberg, Andreas Kamlah und Lothar Schäfer, Hg., Hans Reichenbach und die Berliner Gruppe. Braunschweig: Vieweg, 87-100.

Ash, Mitchell, 2009. Max Wertheimer und Wolfgang Köhler: Gestalttheorie als „dritter Weg“ zwischen Natur- und Geisteswissenschaft. In: Astrid E. Schwarz und Alfred Nordmann, Hg., Das bunte Gewand der Theorie. Vierzehn Begegnungen mit philosophierenden Forschern. Freiburg: Alber, 2009, 263-295.

Benussi, Vittorio, 1904. Zur Psychologie der Gestalterfassung. In: Alexius Meinong, Hg., Untersuchungen zur Gegenstandstheorie und Psychologie, Leipzig: Johann Ambrosius Barth, 303-448.

Benussi, Vittorio, 1914. Die Gestaltwahrnehmungen. Zeitschrift für Psychologie und Physiologie der Sinnesorgane, 69, 256-292.

Bethe, Albrecht, 1928a. Kritische Betrachtungen über den vorklinischen Unterricht. Klinische Wochenschrift, 7, 1481-1483.

Bethe, Albrecht, 1928b. Form und Geschehen im Denken des Heutigen Arztes. Klinische Wochenschrift, 7, 2402-2405.

Bohr, Niels, 1928. Das Quantenpostulat und die neuere Entwicklung der Atomistik. Die Naturwissenschaften, 16, Heft 15, 245-257.

Brod, Max/Weltsch, Felix 1913. Anschauung und Begriff. Grundzüge eines Systems der Begriffsbildung. Leipzig: Kurt Wolff.

Bühler, Karl, 1907. Tatsachen und Probleme zu einer Psychologie der Denkvorgänge. Über Gedanken. Archiv für Psychologie, 9, 297-365. 
Bühler, A., 1916. Beobachtung der Blutbewegung im Auge. Pflügers Archiv für die gesamte Physiologie der Menschen und Thiere, 165, 150-156.

Dąmbska, Izydora, 1978. François Brentano et la Pensée philosophique en Pologne: Casimir Twardowski et son École. Grazer philosophische Studien, 5,117-130.

Dembowski, Jan, 1955 [1946]. Tierpsychologie. Berlin: Akademie.

Ehrenfels, Christian von, 1890. Über Gestaltqualitäten. Vierteljahrsschrift für wissenschaftliche Philosophie, 14, 249-292.

Fitzek, Herbert/Salber, Wilhelm, 1996. Gestaltpsychologie. Geschichte und Praxis. Darmstadt: Wissenschaftliche Buchgesellschaft.

Fleck, Ludwik, [1929] 2011. Zur Krise der „Wirklichkeit“. In: Sylwia Werner und Claus Zittel, Hg., Ludwik Fleck. Denkstile und Tatsachen. Gesammelte Schriften und Zeugnisse. Berlin: Suhrkamp, 52-69.

Fleck, Ludwik/Elster, Olga [1932] 2011. Zur Variabilität der Streptokokken. In: Sylwia Werner und Claus Zittel, Hg., Ludwik Fleck. Denkstile und Tatsachen. Gesammelte Schriften und Zeugnisse. Berlin: Suhrkamp, 126-171.

Fleck, Ludwik [1934] 2011. Wie entstand die Bordet-Wassermann Reaktion und wie entsteht eine wissenschaftliche Entdeckung im allgemeinen? In: Sylwia Werner und Claus Zittel, Hg., Ludwik Fleck. Denkstile und Tatsachen. Gesammelte Schriften und Zeugnisse. Berlin: Suhrkamp, 181-210.

Fleck, Ludwik, [1935] 1980. Entstehung und Entwicklung einer wissenschaftlichen Tatsache. Einführung in die Lehre vom Denkstil und Denkkollektiv. Hg. von Lothar Schäfer und Thomas Schnelle. Frankfurt a. M.: Suhrkamp.

Fleck, Ludwik, [1935] 2011. Über die wissenschaftliche Beobachtung und die Wahrnehmung im allgemeinen. In: Sylwia Werner und Claus Zittel, Hg., Ludwik Fleck. Denkstile und Tatsachen. Gesammelte Schriften und Zeugnisse. Berlin: Suhrkamp, 211-238.

Fleck, Ludwik, [1935] 2011. Zur Frage der Grundlagen des medizinischen Erkennens. In: Sylwia Werner und Claus Zittel, Hg., Ludwik Fleck. Denkstile und Tatsachen. Gesammelte Schriften und Zeugnisse. Berlin: Suhrkamp, 239-259.

Fleck, Ludwik, [1936] 2011. Das Problem einer Theorie des Erkennens. In: Sylwia Werner und Claus Zittel, Hg., Ludwik Fleck. Denkstile und Tatsachen. Gesammelte Schriften und Zeugnisse. Berlin: Suhrkamp, 260-309.

Fleck, Ludwik, [1937] 2011. In der Angelegenheit des Artikels von Frau Izydora Dąmbska in „Przegląd Filozoficzny“ (Jg. 40, Heft III). In: Sylwia Werner und Claus Zittel, Hg., Ludwik Fleck. Denkstile und Tatsachen. Gesammelte Schriften und Zeugnisse. Berlin: Suhrkamp, 320326.

Fleck, Ludwik, [1947] 2011. Schauen, Sehen, Wissen. In: Sylwia Werner und Claus Zittel, Hg., Ludwik Fleck. Denkstile und Tatsachen. Gesammelte Schriften und Zeugnisse. Berlin: Suhrkamp, 390-418.

Fleck, Ludwik, [1948] 2011. Das Problem der wissenschaftlichen Beobachtung. In: Sylwia Werner und Claus Zittel, Hg., Ludwik Fleck. Denkstile und Tatsachen. Gesammelte Schriften und Zeugnisse. Berlin: Suhrkamp, 534-537.

Freudiger, Julie, 2010. Vom „materiellen Lichtbild“ zum „immateriellen Bildlicht“? Josef Albers und James Turrell im Fokus eines wahrnehmungs- und erkenntnistheoretischen Diskurses. Eine vergleichende Werkbetrachtung. Kunsttexte 4, 1-15, http://edoc.hu-berlin.de/ kunsttexte/2010-4/freudiger-julie-8/PDF/freudiger.pdf

Frostig, Jakob, 1929. Das schizophrene Denken. Phänomenologische Studien zum Problem der widersinnigen Sätze. Leipzig: Thieme.

Frostig, Jakob, 1930. Beitrag zur Phänomenologie der autistischen Gestalts- und Wortneubildung. Zeitschrift für die gesamte Neurologie und Psychiatrie, 125, Nr. 1, 700-733.

Gottschaldt, Kurt, 1926. Über den Einfluss der Erfahrung auf die Wahrnehmung von Figuren. Psychologische Forschung, 8, 261-317.

Hagner, Michael, 2010. Sehen, Gestalt und Erkenntnis im Zeitalter der Extreme. Zur historischen Epistemologie von Ludwik Fleck und Michael Polanyi. In: Lena Bader, Martin Gaier und Falk Wolf, Hg., Vergleichendes Sehen. München: Wilhelm Fink, 575-595.

Hagner, Michael, 2012. Perception, Knowledge and Freedom in the Age of Extremes: On the Historical Epistemology of Ludwik Fleck and Michael Polanyi. Studies in East European Thought, 1-2, 107-120. 
Harrington, Anne, 1996. Reenchanted Science: Holism in German Culture from Wilhelm II to Hitler. Princeton: Princeton University Press.

Harrington, Anne, 2002. Die Suche nach Ganzheit. Die Geschichte biologisch-psychologischer Ganzheitslehren. Vom Kaiserreich bis zur New-Age-Bewegung, Reinbek: Rowohlt.

Herrmann, Theo, 1976. Ganzheitspsychologie und Gestalttheorie. In Heinrich Balmer, Hg., Die Psychologie des 20. Jahrhunderts. Bd. 1: Die europäische Tradition. Zürich: Kindler, 573-658.

Hornbostel, Erich von, 1922. Über optische Inversion. Psychologische Forschung, 1, 130-156.

Ingarden, Roman, [1947/1948] 1964/1965. Der Streit um die Existenz der Welt. Tübingen: Niemeyer.

Ingarden, Roman, [1937/1964] 1976. Gegenstand und Aufgaben der Literaturwissenschaft. Aufsätze aus den Jahren 1937-1964. Tübingen: Niemeyer.

Lévy-Bruhl, Lucien, 1921 [1. Aufl.]/1926 [2. Aufl.] [fr. 1918]. Das Denken der Naturvölker. Wien: Braumüller.

Löwy, Ilana, 2008. Ways of Seeing: Ludwik Fleck and Polish Debates on the Perception of Reality, 1890-1947. Studies in History and Philosophy of Science, 39, 375-383.

Koffka, Kurt, 1914. Psychologie der Wahrnehmung. Die Geisteswissenschaften, 1, 711-716, 796800.

Koffka, Kurt, 1919a. Zur Grundlegung der Wahrnehmungspsychologie. Eine Auseinandersetzung mit V. Benussi. Zeitschrift für Psychologie, 73, 11-19.

Koffka, Kurt, 1919b. Über den Einfluss der Erfahrung auf die Wahrnehmung. Die Naturwissenschaften, 7, 597-605.

Koffka, Kurt, 1925. Introspection and the Method of Psychology. British Journal of Psychology, 15, 149-161.

Koffka, Kurt, 1929. Die Wahrnehmung von Bewegung. In: Albrecht Bethe, Gustav von Bergmann, Gustav Embden, Alexander Ellinger, Hg., Handbuch normaler und pathologischer Physiologie. Bd. 2: Rezeptionsorgane. Berlin: Julius Springer, 1166-1214.

Köhler, Wolfgang, 1913. Über unbemerkte Empfindungen und Urteilstäuschungen. Zeitschrift für Psychologie und Physiologie der Sinnesorgane, 66, 51-80.

Köhler, Wolfgang, 1917. Intelligenzprüfungen an Anthropoiden. Berlin: Akademie der Wissenschaften.

Köhler, Wolfgang, 1920. Die physischen Gestalten in Ruhe und im stationären Zustand. Eine naturphilosophische Untersuchung. Braunschweig: Vieweg.

Köhler, Wolfgang, 1928. Bemerkungen zur Gestalttheorie. Psychologische Forschung, 11, 188-234.

Meinong, Alexius, 1929. Abhandlungen zur Psychologie. Bd. 1. Leipzig: J. A. Barth.

Meinong, Alexius [1889] 1968/1978. Ueber Phantasie-Vorstellung und Phantasie. In: Roderick M. Chisholm, Rudolf Kindinger und Rudolf Haller, Hg., Alexius Meinong. Gesamtausgabe. Graz: Akademische Druck- und Verlagsanstalt, Bd. 1 (1968), 109-192.

Metzger, Wolfgang, 1929. Psychologische Mitteilungen. Die Naturwissenschaften, 45, 843-848.

Petersen, Hans, 1928. Über die Rolle der Anatomie im Lehrgang des künftigen Arztes. Klinische Wochenschrift, 7, 1872-1875.

Petersen, Hans, 1936. Ludwik Flecks Lehre vom Denkstil und dem Denkkollektiv. Klinische Wochenschrift, 15, 239-242.

Pijper, A., 1930. Begeißelung von Typhus- und Proteusbazillen. Zentralblatt für Bakteriologie, Parasitenkunde, Infektionskrankheiten und Hygiene, Serie I. Originale, 118, 113-121.

Pijper, A., 1931. Nochmals über die Begeißelung von Typhus- und Proteusbazillen. Zentralblatt für Bakteriologie, Parasitenkunde, Infektionskrankheiten und Hygiene, Serie I. Originale, 123, 195-201.

Pijper, A., 1938. Dark-Ground Studies of Flagellar and Somatic Agglutination of B. Typhosus. Journal of Pathology and Bacteriology, 97, 1-17.

Rorschach, Hermann, 1921. Psychodiagnostik. Methodik und Ergebnisse eines wahrnehmungsdiagnostischen Experiments (Deutenlassen von Zufallsformen). Bern: Bircher.

Rubin, Edgar, 1921. Visuell wahrgenommene Figuren. Studien in psychologischer Analyse. Kopenhagen: Gyldendal.

Sander, Friedrich, 1928. Experimentelle Ergebnisse der Gestaltpsychologie. E. Becher, Hg., X. Kongress experimentelle Psychologie. Jena: Gustav Fischer.

Simons, Oliver, 2007. Raumgeschichten. Topographien der Moderne in Philosophie, Wissenschaft und Literatur. München: Wilhelm Fink.

Smith, Barry, 1988a, Hg., Foundations of Gestalt Theory. München: Philosophia. 
Smith, Barry, 1988b. Gestalt Theory: An Essay in Philosophy. In: ders., Hg., Foundations of Gestalt Theory. München: Philosophia, 11-81.

Smith, Barry, 1994. Austrian Philosophy. The Legacy of Franz Brentano. Chicago/LaSalle, Illinois: Open Court Publishing Company.

Stachel, Peter, 1999. Leibniz, Bolzano und die Folgen. Zum Denkstil der österreichischen Philosophie, Geistes- und Sozialwissenschaften. In: Karl Acham, Hg., Geschichte der österreichischen Humanwissenschaften. Bd. 1, Wien: Passagen, 253-296.

Stahnisch, Frank/Bauer, Heijko, Hg., 2007: Bild und Gestalt: Wie formen Medienpraktiken das Wissen in Medizin und Humanwissenschaften? Münster: LIT.

Stock, Mechthild/Stock, Wolfgang, 2008, Hg., Psychologie und Philosophie der Grazer Schule. Amsterdam: Rodopi.

Sudhoff, Karl, 1907. Tradition und Naturbeobachtung in den Illustrationen medizinischer Handschriften und Frühdrucke vornehmlich des 15. Jahrhunderts. Leipzig: J. A. Barth.

Uexküll, Jakob von, 1920. Theoretische Biologie. Berlin: Verlag Gebrüder Paetel.

Zittel, Claus, 2010. The Politics of Cognition. Genesis and Development of Ludwik Fleck's Comparative Epistemology. In: Moritz Epple und Claus Zittel, Hg., Science as Cultural Practice. Vol. 1.: Cultures and Politics of Research from the Early Modern Period to the Age of Extremes. Wissenskultur und gesellschaftlicher Wandel. Berlin: Akademie-Verlag, 183-200.

Zittel, Claus, 2011. Ludwik Fleck und der Stilbegriff in den Naturwissenschaften. Stil als wissenschaftshistorische, epistemologische und ästhetische Kategorie. In: Horst Bredekamp und John Krois, Hg., Sehen und Handeln. Berlin: Akademie, 171-206.

Zittel, Claus, 2012. Ludwik Fleck and the Concept of Style in the Natural Sciences. Studies in East European Thought, 1-2, 53-79.

\section{Claus Zittel}

Institut für Deutsche und Niederländische Philologie

Freie Universität Berlin

Habelschwerdter Allee 45

14195 Berlin

Deutschland

E-Mail: zittel@zedat.fu-berlin.de 